\title{
Identification of a mitochondria-targeting fluorescent small molecule for dual phototherapy
}

\author{
Jie Chen, Xu Tan, Shenglin Luo, Lei Long, Lang Liu, Zujuan Liu, \\ $\mathrm{Yu}$ Wang* and Chunmeng $\mathrm{Shi}^{\dagger}$ \\ State Key Laboratory of Trauma, Burns and Combined Injury \\ Institute of Combined Injury \\ Chongqing Engineering Research Center for Nanomedicine \\ College of Preventive Medicine \\ Army Medical University, 30, Gaotanyan Street \\ Chongqing 400038, P. R. China \\ *wangyusmmu@163.com \\ ${ }^{\dagger}$ shicm@sina.com
}

Received 4 December 2017

Accepted 22 February 2018

Published 2 April 2018

\begin{abstract}
Phototherapy, mainly including photodynamic therapy (PDT) and photothermal therapy (PTT), is a noninvasive and effective approach for cancer treatment. Since integration of PDT and PTT for simultaneous synergistic PDT/PTT treatment enables us to improve phototherapeutic efficacy significantly, it has been attracting a lot of investigations in current days. Here, we introduce IR-52, a new mitochondria-targeting near infrared (NIR) fluorescent small molecule, which possesses structure-inherent PTT and PDT synergistic phototherapeutic effects without conjugation to specific ligands. After NIR light irradiation $\left(808 \mathrm{~nm}, 2 \mathrm{~W} / \mathrm{cm}^{2}\right.$, $5 \mathrm{~min}$ ), both the hyperthermia and excessive singlet oxygen levels were determined when dissolving IR-52 in aqueous solutions. In vitro photoinduced cytotoxicity studies showed significant lower cell viabilities and higher necrotic/apoptotic rates when cancer cells were treated with IR-52 and irradiation, and its' mitochondrial localization in cancer cells would partially explain its high cytotoxicity. Further in vivo synergetic PDT and PTT effects were demonstrated by high tumor surface temperature and significant inhibition of tumor growth. Our results strongly suggest that IR-52, which possesses excellent photosensitivity, may provide a promising strategy for tumor treatment with decreased side effects.
\end{abstract}

Keywords: Photosensitizer; photodynamic therapy; photothermal therapy; mitochondria.

$\dagger$ Corresponding author.

This is an Open Access article published by World Scientific Publishing Company. It is distributed under the terms of the Creative Commons Attribution 4.0 (CC-BY) License. Further distribution of this work is permitted, provided the original work is properly cited. 


\section{Introduction}

Phototherapy has become one of the main means for cancer treatment available in clinic following with surgical treatment, chemotherapy and radiotherapy. This type of light-triggered treatment has remarkably improved selectivity and reduced the side effects as compared to conventional therapies. ${ }^{1-3}$ Phototherapy mainly includes photodynamic therapy (PDT) and photothermal therapy (PTT). ${ }^{4,5}$ For PDT or PTT, the key factor to determine their therapeutic effects is the photosensitizer (PS) ${ }^{6-8} \mathrm{~A}$ desirable PS should have superior photosensitivity to ensure the phototherapeutic effect and high specificity to reduce the side effects. ${ }^{9-11}$ The most common way to enhance the photosensitivity is to synthesis a photothermal PS and a photodynamic PS together as reported in many nanomaterials. ${ }^{12,13}$ The typical way to improve the specificity is to conjugate the PSs with a target ligand, which can bind to the cancer cells overexpressed biomarkers efficiently. ${ }^{14,15}$ However, there are still many problems for these nanomaterial-based multifunctional PSs, such as poor tumor-targeting activity, unsatisfied high-quality control during large-scale preparation, potential long-term toxicity concerns, which lead to the difficulties for further clinical application. ${ }^{16,17}$

Indocyanine green (ICG), a water-soluble anionic tricarbocyanine dye, which can simultaneously achieve PTT and PDT effects under single wavelength near infrared (NIR) laser without conjunction, ${ }^{18,19}$ is the only Food and Drug Administration (FDA) approved NIR agent. However, ICG therapeutic effect is limited by various factors, such as poor in vitro aqueous stability, rapid body clearance and lack of targeting property. Recently, some new NIR fluorophores, with ICG as the prototype structure, have been reported to improve the therapeutic effect and targeting ability, but few of them have both PDT and PTT effects.

In the present study, we developed a lipophilic cationic heptamethine dye, IR-52, with a peak optical absorption of $789 \mathrm{~nm}$ wavelength, which can preferentially accumulate at tumor site and further retain in the mitochondria of cancer cells and is excited with $808 \mathrm{~nm}$ laser to generate potent local heat and singlet oxygen, thus destroying cancer cells both in vitro and in vivo via its simultaneous synergistic PDT/PTT property. As expected, a multifunctional small molecule simultaneously with properties of cancer targeting, NIR imaging, PDT and PTT synergistic phototherapy was finally obtained. This PS possesses superior photosensitivity, and is expected to improve the phototherapeutic effect with decreased side toxicity and may provide a new practical route for cancer treatment.

\section{Materials and Methods}

\subsection{Cell lines}

Human lung cancer cell lines A549 and mouse Lewis lung carcinoma (LLC) cells were purchased from American Type Culture Collection (ATCC, Manassas, VA, USA) and cultured in ATCC recommended medium (Hyclone, Logan, Utah, USA), supplemented with $10 \%$ fetal bovine serum (FBS, Gibco, Grand Island, USA), $1 \%$ penicillin and streptomycin (Beyoyime, Shanghai, China). Cells were incubated under $5 \% \mathrm{CO}_{2}$ at $37^{\circ} \mathrm{C}$ and were passed regularly and subcultured to $\sim 80 / 90 \%$ of confluence before all the experiments.

\subsection{Singlet oxygen detection}

To evaluate the generation of singlet oxygen from samples, IR-52 $(10 \mu \mathrm{M}$, dissolved in water $)$ and singlet oxygen sensor green (SOSG) $(1 \mu \mathrm{M}$, dissolved in water containing $2 \%$ methanol) were mixed. NIR fluorescence spectrometer was used to obtain the fluorescence intensity after $5 \mathrm{~min}$ irradiation $\left(2 \mathrm{~W} / \mathrm{cm}^{2}\right)$.

\subsection{Mitochondrial localization}

A549 cells were cultured in a $35 \mathrm{~mm}$ petri-dish overnight and incubated with vehicle control or $10 \mu \mathrm{M}$ IR-52 for $15 \mathrm{~min}$ at $37^{\circ} \mathrm{C}$, and then stained with mito-tracker green for $10 \mathrm{~min}$ (Lyso Tracker Green was used as a negative control). Dark condition was limited strictly during the whole procedures. The fluorescence of cells was obtained by the confocal microscope.

\subsection{In vitro phototherapy}

A549 and LLC were used to evaluate the phototherapy effect of IR-52 in vitro. Cells were incubated with different concentrations $(0,0.75,1.5,3,6$ and $12 \mu \mathrm{M})$ of IR-52 for $24 \mathrm{~h}$ before laser irradiation $\left(2 \mathrm{~W} / \mathrm{cm}^{2}\right)$ for $5 \mathrm{~min}$ or not. To investigate the 
single PDT effect or PTT effect, cells were treated with $10 \mathrm{mM}$ NAC to avoid producing singlet oxygen or maintained at the constant temperature $\left(37^{\circ} \mathrm{C}\right)$ to avoid hyperthermia generation, respectively, during laser irradiation. The relative cell viabilities were detected by CCK- 8 assay after another $24 \mathrm{~h}$ incubation.

The synergistic PDT/PTT effects of IR-52 were further confirmed by calcein $\mathrm{AM}$ and propidium iodide (PI), PI and Annexin V co-staining. Cells were incubated with IR-52 before irradiation by an NIR $(808 \mathrm{~nm})$ laser, and then incubated with $2 \mu \mathrm{M}$ calcein AM and $2 \mu \mathrm{M}$ PI $24 \mathrm{~h}$ later. Fluorescent images were obtained by a biological inverted microscope. The apoptosis of different groups was detected according to the direction of Annexin $\mathrm{V}$ FITC/PI kit.

\subsection{Animals and tumor model}

All C57BL/6 mice were purchased from the laboratory animal center of the Army Medical University. Animal protocols were according to the "Animal Care and Use Committee Guidelines of the Army Medical University". LLC tumor xenograft models were established by subcutaneous injection of $1 \times 10^{6}$ LLC cells onto the hind legs of mice (5-6 weeks old). $1 \times 10^{6}$ LLC cells suspended in $50 \mu \mathrm{L}$ PBS were injected into the subcutaneous using a 28-gauge needle.

\subsection{In vivo NIR fluorescence and thermal imaging}

In all subcutaneous LLC tumor xenograft models, the animals were injected with IR-52 at the dosage of $0.4 \mathrm{mg} / \mathrm{kg}$ via the tail vein to gain the NIR imaging, and at a dosage of $5 \mathrm{mg} / \mathrm{kg}$ to gain the thermal imaging when the tumors' volume reached about $150 \mathrm{~mm}^{3}$ (to avoid the situation that tumors' size is too large to be covered completely by laser irradiation).

\subsection{In vivo synthetic PDT and PTT effects}

LLC tumor xenograft model was used to investigate the synthetic PDT and PTT effect of IR-52 in vivo. Twenty mice were divided into three groups: PBS injection group, IR-52 $(5 \mathrm{mg} / \mathrm{kg}$ IR-52 diluted in
$200 \mathrm{~mL}$ PBS) injection group and IR-52 combined with NIR laser irradiation group (1-2 mice from each group were used for histomorphology tests). Irradiation groups were irradiated by $808 \mathrm{~nm}$ laser at the power density of $0.8 \mathrm{~W} / \mathrm{cm}^{2}$ for $5 \mathrm{~min}, 24 \mathrm{~h}$ post-injection. Tumor volumes and body weights of mice were measured during the procedure (tumor volume $=$ length $\left.\times(\text { width })^{2} / 2\right)$.

\section{Results and Discussion}

\subsection{Structure and optical properties of IR-52}

The chemical structure of IR-52 was shown in Fig. 1. The detailed synthesis process of IR-52 was according to our previous work. ${ }^{20}$ The absorption and fluorescence spectra of IR-52 were investigated in methanol (MeOH) and 10\% FBS (Figs. 2(a)-2(d)). Compared to ICG, which is the prototype structure of our new NIR fluorophore and already has been a clinically available heptamethine dye, IR-52 has the maximum absorbance and emission wavelength near $780 \mathrm{~nm}$ and $800 \mathrm{~nm}$, which were in the NIR region $(700-900 \mathrm{~nm})$. Notably, IR-52 has higher absorption and fluorescence intensity than ICG, which would decrease tissue absorption/autofluorescence, and improve the fluorescence signal contrast.

To investigate the PDT efficacy of IR-52, we detect the generation of singlet oxygen $\left({ }^{1} \mathrm{O}_{2}\right)$ by SOSG. SOSG was added into $10 \mu \mathrm{M}$ IR-52 aqueous solution and irradiated with $808 \mathrm{~nm}$ laser $\left(2 \mathrm{~W} / \mathrm{cm}^{2}\right.$, $5 \mathrm{~min})$. The fluorescence intensity of SOSG in IR-52 was remarkably higher than blank water (Fig. 3(a)). To investigate the PTT efficacy, we tested the temperature change of IR-52. Encouragingly, the temperature was found to increase sharply near about $30^{\circ} \mathrm{C}$ (Fig. 3(b)), which is high enough to cause the death of tumor cells. In contrast, the temperature increased moderately

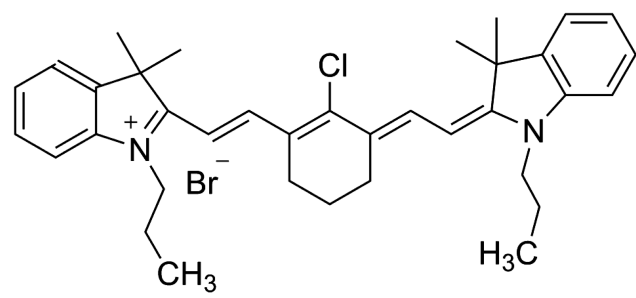

Fig. 1. Chemical structure of IR-52. 


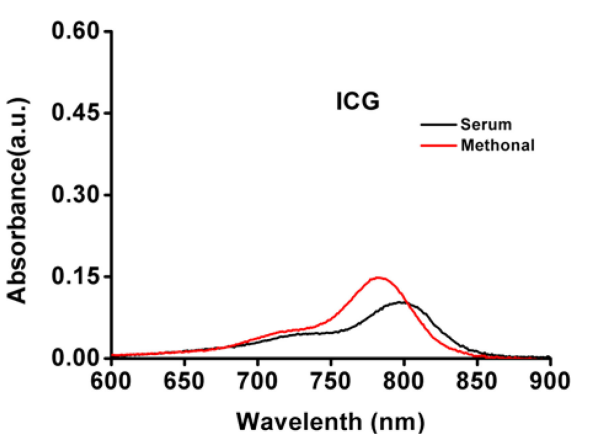

(a)

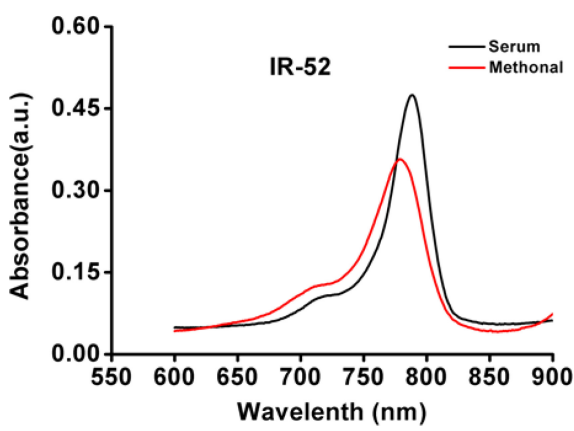

(c)

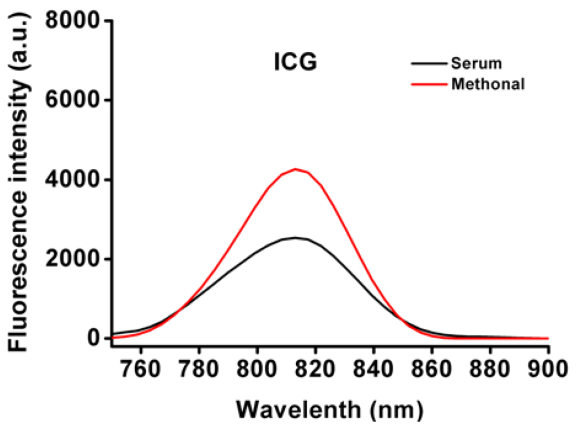

(b)

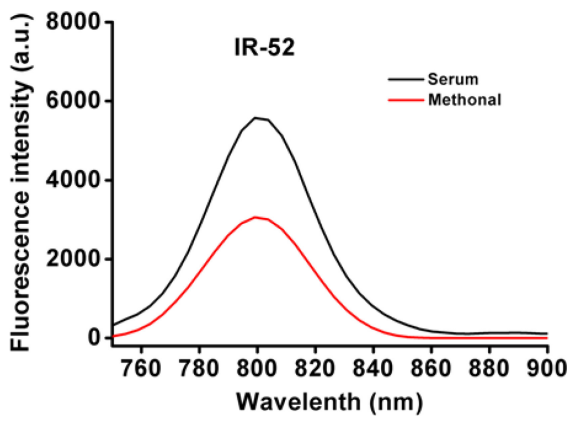

(d)

Fig. 2. The absorption and fluorescence spectra of IR-52 compared to ICG.

(less than $20^{\circ} \mathrm{C}$ ) for ICG. Therefore, IR-52 is a more excellent potent PS than ICG.

\subsection{In vitro phototherapy of IR-52}

The in vitro phototherapeutic effect of IR-52 was evaluated on A549 and LLC. Cells were exposed to $808 \mathrm{~nm}$ laser irradiation $\left(2 \mathrm{~W} / \mathrm{cm}^{2}\right)$ after incubation with different concentrations of IR-52 (ranging from $0 \mu \mathrm{M}$ to $12 \mu \mathrm{M})$ for $24 \mathrm{~h}$. The relative cell viabilities were determined by the CCK- 8 assay after another

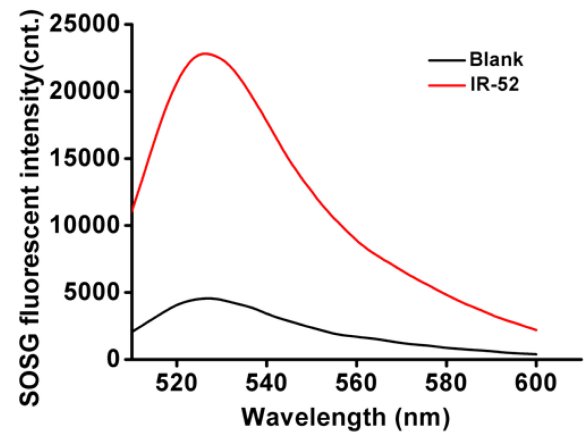

(a)
$24 \mathrm{~h}$ incubation (Figs. 4(a) and 4(b)). The results showed that the cell viability of A549 (IR-52 irradiation group) exhibited a remarkable decrease with the increased concentration. More cytotoxicity on LLC cells was shown at the concentration of $12 \mu \mathrm{M}$, compared to the nonirradiation group. Calcein AM and PI co-staining were carried out on A549 cells to visualize live cells or dead/late apoptotic cells, respectively, to further confirm the synergistic PDT and PTT effects of IR-52 (Fig. 4(c)). The results showed that neither IR-52 nor laser alone has much

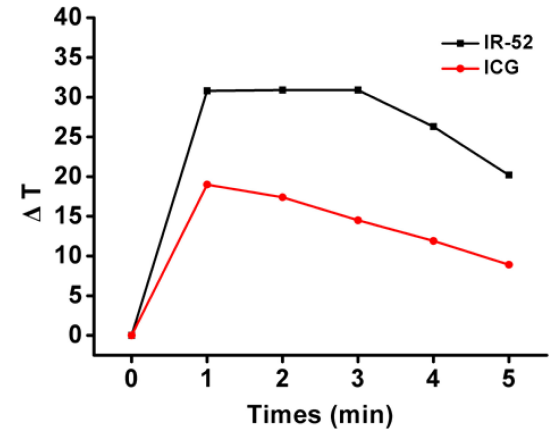

(b)

Fig. 3. (a) Singlet oxygen generation and (b) temperature change curves of IR-52 compared with ICG during a single NIR laser irradiation $\left(808 \mathrm{~nm}, 1.5 \mathrm{~W} / \mathrm{cm}^{2}\right)$. 

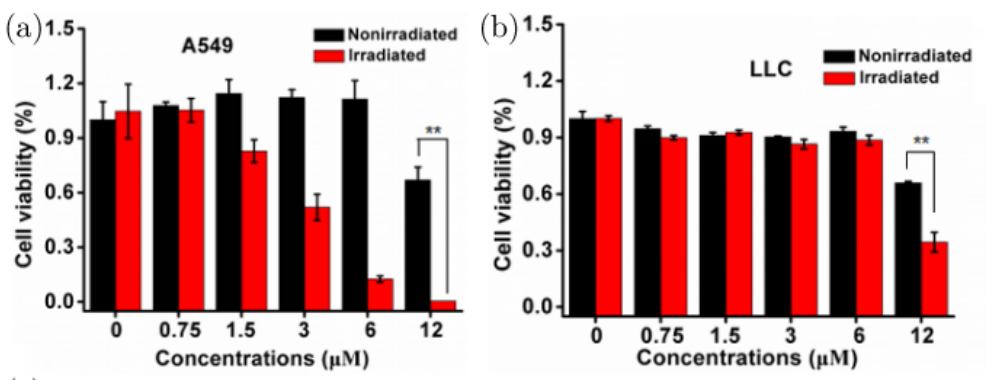

(d)
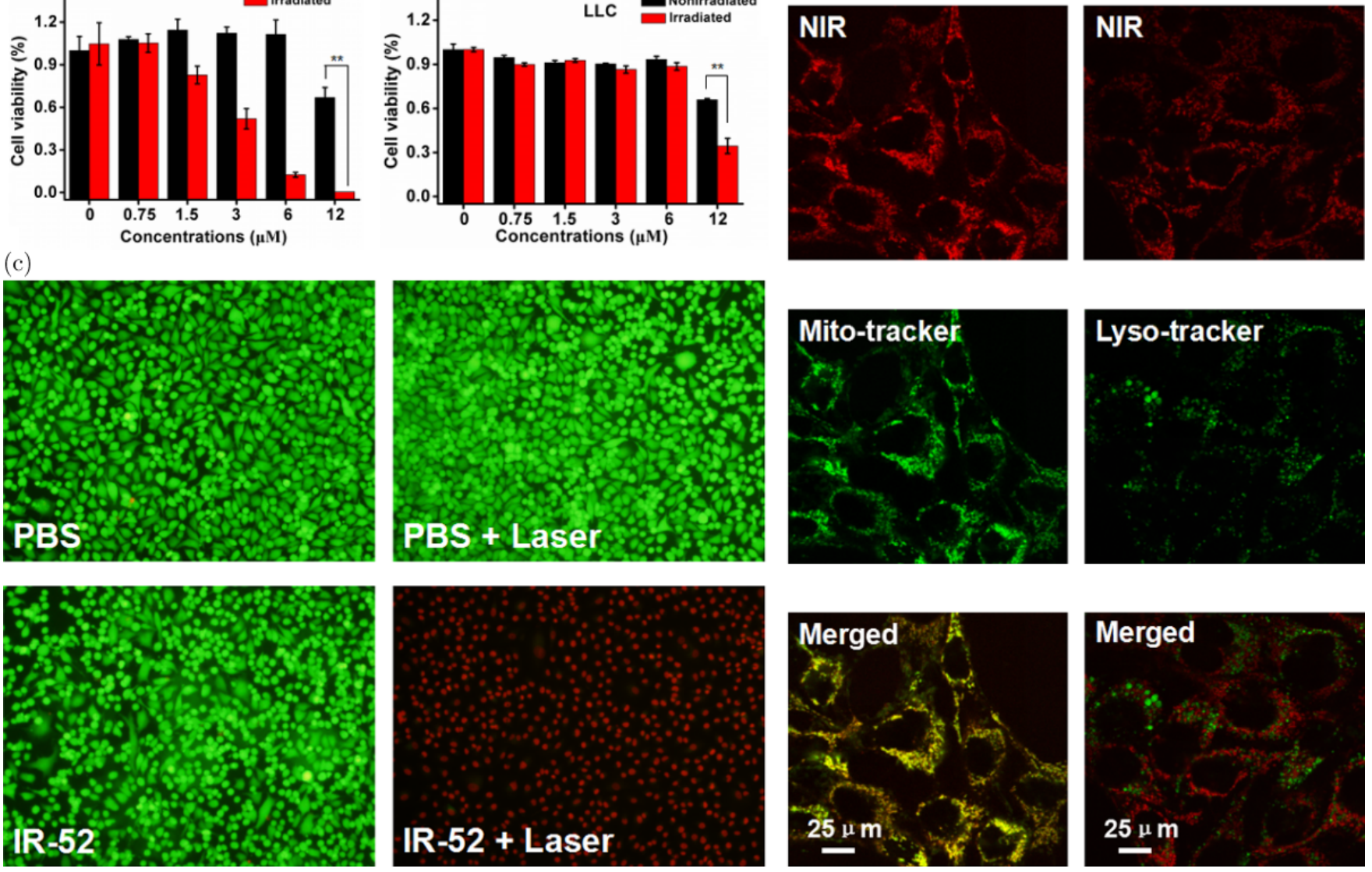

Fig. 4. In vitro cytotoxicity induced by phototherapy and mitochondrial localization. (a, b) Viabilities of A549 and LLC cells after incubated with different concentrations (ranging from $0 \mu \mathrm{M}$ to $12 \mu \mathrm{M}$ ) of IR-52 for $24 \mathrm{~h}$ exposed to laser irradiation (808 $\mathrm{nm}, 2 \mathrm{~W} /$ $\mathrm{cm}^{2}$ ) or not for $5 \mathrm{~min}$. Those cells were re-incubated for $24 \mathrm{~h}$ before CCK- 8 assay was conducted. (c) Fluorescent images of Calcein AM/PI co-stained A549 cells after different treatments (Green: Calcein AM, Red: PI). (d) Mitochondrial localization was determined by co-stained IR-52 with Mito-tracker in A549 cells. Lyso Tracker Green was used as a negative control.

virulent influence on cells, but cells pretreated with IR-52 and then 5 min light irradiation showed nearly no viable cells.

A549 cells were co-stained with IR-52 and the mitochondrial tracker (or Lyso Tracker) to investigate the mitochondrial localization (Fig. 4(d)). Pearson's correlation coefficient and overlap coefficient were quantitatively determined using Leica Mircosystems LAS AF-TCS SP5 software. Pearson's correlation coefficient of IR-52 and MitoTracker in A549 cells has been determined with 0.8155 , while overlap coefficient was 0.963 , respectively. These results showed that IR-52 accumulated mostly in the mitochondria of cancer cell. These results would partially explain the high photoinduced cytotoxicity of IR-52. We also costained IR-52 with Lyso Tracker Green as a negative control experiment, as expected that, nearly no overlap between red fluorescence of IR-52 and green fluorescence of Lyso Tracker Green. To investigate whether the cell death undergone the apoptosis or necrosis, cells were labeled with PI and Annexin V (Annexin V-FITC). Figure 5 shows that nearly no apoptosis was observed in the cells treated with IR52 or light irradiation alone, as well as the blank control. In contrast, cells pretreated with IR-52 and then 5 min light irradiation exhibited obvious apoptosis rate. Those results indicated that IR-52 exhibited effective light-induced cancer cell death.

\subsection{In vivo phototherapy of IR-52}

Encouraged by the results in vitro, we further studied the anticancer efficacy of IR-52 on animal models. C57 mouse bearing LLC was established for IR-52 injection to confirm the tumor preferential 


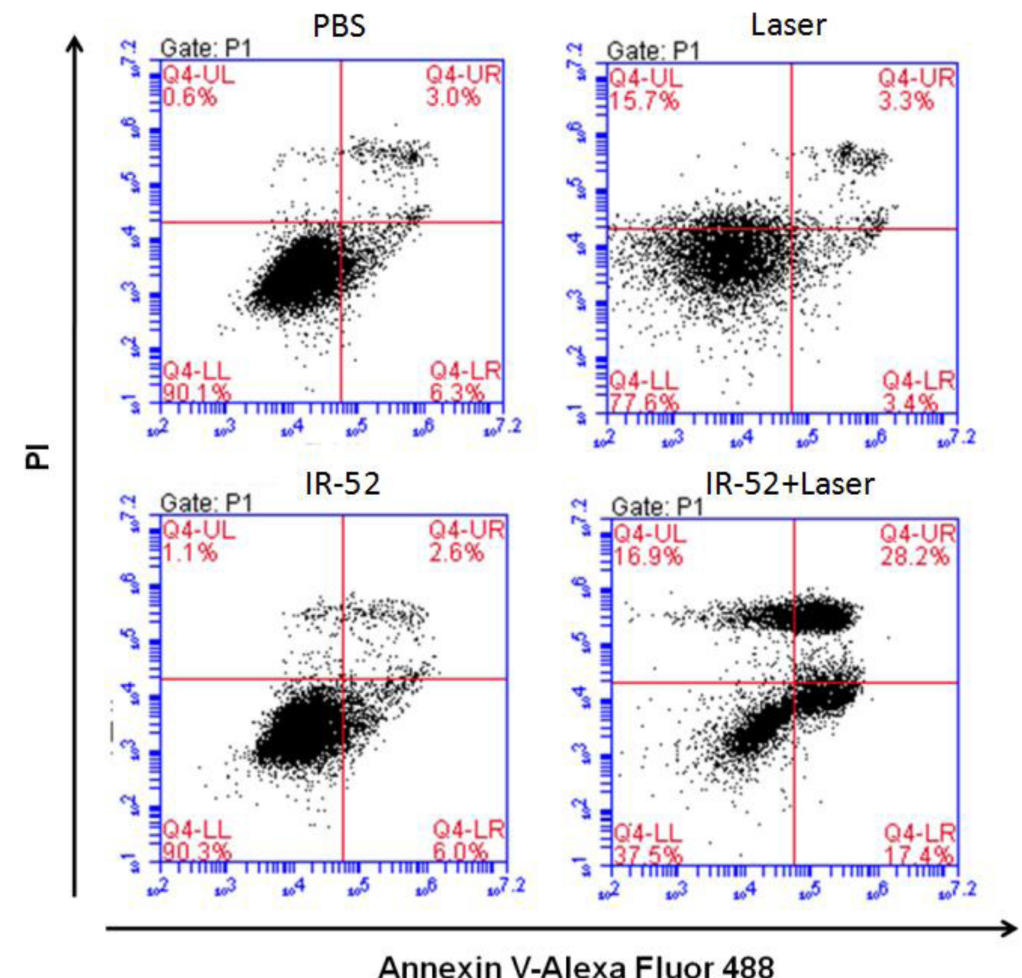

Fig. 5. Apoptosis assay in vitro: A549 cells were assessed for apoptosis with PI and Annexin V-Alexa Fluor 488 staining by flow cytometry after different treatments.

(a)

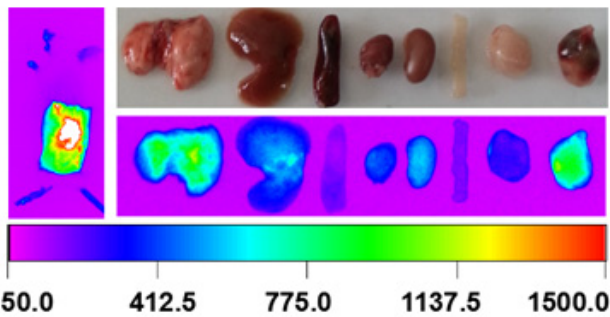

(b)

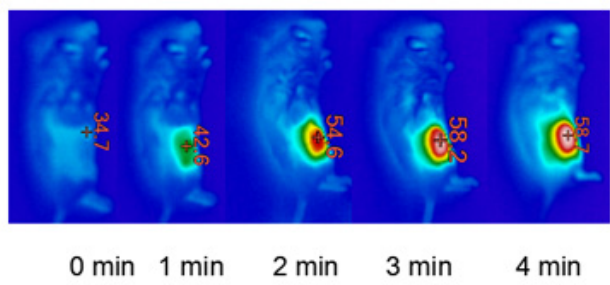

(e)

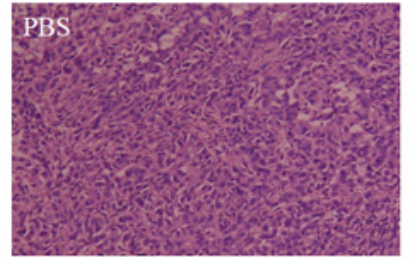

(c)

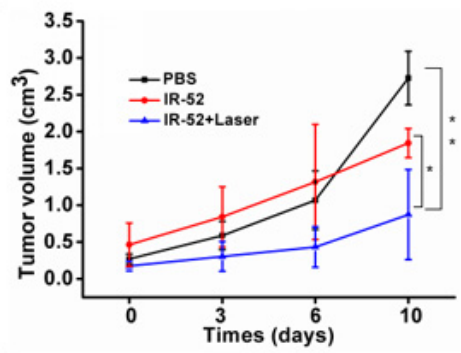

(d)
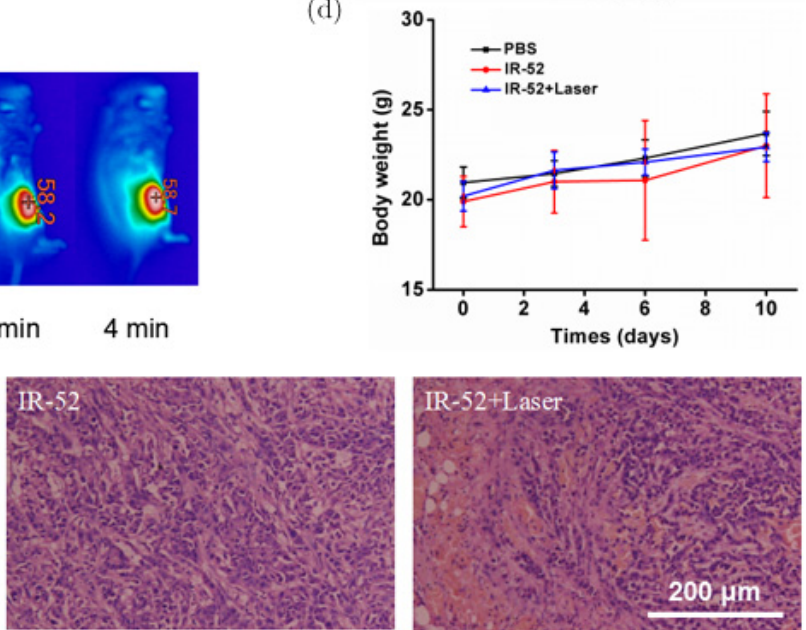

Fig. 6. Cancer targeting and phototherapy in vivo. (a) NIR fluorescent images of whole body and ex vivo main organs (dissected at $72 \mathrm{~h}$ ) and (b) IR thermal images were detected on subcutaneous LLC tumor xenograft mice. (c) Tumor growth curves and (d) body weights of mice in different groups were measured during the 10-day evaluation period. The results were expressed as the mean \pm s.d. $\left(n=5,{ }^{*} p<0.05,{ }^{* *} p<0.01\right)$. (e) H\&E staining of tumors $24 \mathrm{~h}$ post-irradiation. 
accumulation (Fig. 6(a)). The mice were injected with IR-52 at a dose of $0.3 \mathrm{mg} / \mathrm{kg}$ via the tail vein. NIR fluorescent images showed that the intense fluorescence signals associated with the implanted tumors can be clearly visualized after IR-52 injection $24 \mathrm{~h}$, and there is low background interfering fluorescence. Meanwhile, its targeted imaging property would make tumors with the margins clearly visualized, and provide the potential for precisely imaging-guided cancer phototherapy, which is helpful in minimizing the treatment-related side effects.

Figure 6(b) shows that the real-time tumor surface temperature of the mice in the irradiation group amazingly increased to about $59^{\circ} \mathrm{C}$, which was confirmed to be high enough to lead the death of cancer cells. LLC tumor-bearing models were established and divided into three groups with five mice per group: PBS injection group, IR-52 injection group and IR-52 injection combined with NIR laser irradiation group. Each group was given $200 \mu \mathrm{L}$ PBS solution or $5 \mathrm{mg} / \mathrm{kg}$ IR-52 diluted in $200 \mu \mathrm{L}$ PBS solution, respectively. Tumor growth was monitored every 3-4 days after treatments. Remarkably, tumors treated with IR-52 plus laser irradiation showed significant inhibition of growth of tumor during the whole treatment up to 10 days. However, the tumors treated with IR-52 and PBS without laser irradiation grew rapidly (Fig. 6(c), $\left.n=5,{ }^{*} p<0.05,{ }^{* *} p<0.01\right)$. Figure $6(\mathrm{~d})$ shows no significant difference in all the groups on body weight, which implies a desirable safety in IR-52 treated mice. Figure 6(e) shows that after H\&E staining of these tumors $24 \mathrm{~h}$ post-irradiation, large areas of severe tissue damage including apoptosis and necrosis were observed only in tumors of IR-52 plus irradiation group. In summary, IR-52 is effective and a potential way for the treatment of cancer.

\section{Conclusion}

In this study, we obtained a small molecule that simultaneously exhibits tumor-targeted imaging and treatment, integrating diagnostics and therapeutics by virtue of the molecules' inherent chemical structures. IR-52 was successfully developed for in vitro and in vivo $\mathrm{PDT}$ and $\mathrm{PTT}$ combination therapy for cancer. Although it was confirmed that the fluorophore was localized at the mitochondria, the detailed mechanism still needs to be further studied. In summary, a small organic molecule simultaneously with cancer cell mitochondrial targeting, NIR imaging and synchronous PDT/PTT effects was developed in this report. This small molecule PS, with excellent photosensitivity, is expected to improve the phototherapeutic effect with decreased side toxicity and may provide a practical way for cancer treatment.

\section{Acknowledgments}

This work is supported by the National Natural Science Foundation of China (Grant No. 81402784) and University Innovation Team Building Program of Chongqing (CXTDG201602020). Yu Wang is a co-corresponding author in this work.

\section{References}

1. S. L. Luo, Z. Y. Yang, X. Tan, Y. Wang, Y. P. Zeng, Y. Wang, C. M. Li and R. Li, "Multifunctional photosensitizer grafted on polyethylene glycol and polyethylenimine dual-functionalized nanographene oxide for cancer-targeted near-infrared imaging and synergistic phototherapy," ACS. Appl. Mater. Interfaces 8, 17176-17186 (2016).

2. N. Li, T. T. Li, C. Hu, X. M. Lei, Y. P. Zuo and H. Y. Han, "Targeted near-infrared fluorescent turn-on nanoprobe for activatable imaging and effective phototherapy of cancer cells," ACS. Appl. Mater. Interfaces 8, 15013-15023 (2016).

3. R. R. Guo, H. B. Peng, Y. Tian, S. Shen and W. L. Yang, "Mitochondria-targeting magnetic composite nanoparticles for enhanced phototherapy of cancer," Small 12, 4541-4552 (2016).

4. Z. H. Sheng, D. H. Hu, M. B. Zheng, P. F. Zhao, H. L. Liu, D. Y. Gao, P. Gong, G. H. Gao, P. F. Zhang, Y. F. Ma and L. T. Cai, "Smart human serum albumin-indocyanine green nanoparticles generated by programmed assembly for dual-modal imaging-guided cancer synergistic phototherapy," ACS. Nano 8, 12310-12322 (2014).

5. R. Chen, X. Wang, X. K. Yao, X. C. Zheng, J. Wang and X. Q. Jiang, "Near-IR-triggered photothermal/photodynamic dual-modality therapy system via chitosan hybrid nanosphere," Biomaterials 34, 8314-8322 (2013).

6. J. P. Celli, B. Q. Spring, I. Rizvi, C. L. Evans, K. S. Samkoe, S. Verma, B. W. Pogue and T. Hasan, "Imaging and photodynamic therapy: Mechanisms, monitoring, and optimization," Chem. Rev. 110, 2795-2838 (2010). 
7. E. Boisselier and D. Astruc, "Gold nanoparticles in nanomedicine: Preparations, imaging, diagnostics, therapies and toxicity," Chem. Soc. Rev. 38, 1759-1782 (2009).

8. E. J. Sanchez-Barcelo and M. D. Mediavilla, "Recent patents on light-based therapies: Photodynamic therapy, photothermal therapy and photoimmunotherapy," Recent. Pat. Endocr. Metab. Immune Drug Discov. 8, 1-8 (2014).

9. L. W. Lin, L. Xiong, Y. Wen, S. L. Lei, X. F. Deng, Z. P. Liu, W. Chen and X. G. Miao, "Active targeting of nano-photosensitizer delivery systems for photodynamic therapy of cancer stem cells," J. Biomed. Nanotechnol. 11, 531-554 (2015).

10. T. G. St Denis and M. R. Hamblin, "Synthesis, bioanalysis and biodistribution of photosensitizer conjugates for photodynamic therapy," Bioanalysis 5, 1099-1114 (2013).

11. N. V. Kudinova and T. T. Berezov, "Photodynamic therapy: Search for ideal photosensitizer," Biomed. Khim. 55, 558-569 (2009).

12. C. W. Chung, C. W. Choi, Y. I. Jeong and D. H. Kang, "Nano-self assembled photosensitizer composed of methoxy poly(ethylene glycol)-conjugated chlorin e6 for enhanced photosensing of HCT116 cells," J. Nanosci. Nanotechnol. 16, 1379-1383 (2016).

13. W. X. Hou, X. Zhao, X. Q. Qian, F. Pan, C. L. Zhang, Y. M. Yang, J. M. de la Fuente and D. X. Cui, "pH-sensitive self-assembling nanoparticles for tumor near-infrared fluorescence imaging and chemo-photodynamic combination therapy," Nanoscale 8, 104-116 (2016).

14. I. E. Furre, S. Shahzidi, Z. Luksiene, M. T. Møller, E. Borgen, J. Morgan, K. Tkacz-Stachowska, J. M. Nesland and Q. Peng, "Targeting PBR by hexaminolevulinate-mediated photodynamic therapy induces apoptosis through translocation of apoptosis-inducing factor in human leukemia cells," Cancer. Res. 65, 11051-11060 (2005).

15. Z. C. Fan, X. J. Cui, D. Wei, W. Liu, B. H. Li, H. He, H. M. Ye, N. S. Zhu and X. B. Wei, "eEF1A1 binds and enriches protoporphyrin IX in cancer cells in 5-aminolevulinic acid-based photodynamic therapy," Sci. Rep. 6, 25353 (2016).

16. W. B. Wu, D. Mao, F. Hu, S. D. Xu, C. Chen, C. J. Zhang, X. M. Cheng, Y. Y. Yuan, D. Ding, D. L. Kong and B. Liu, "A highly efficient and photostable photosensitizer with near-infrared aggregation-induced emission for image-guided photodynamic anticancer therapy," Adv. Mater. 29 (2017).

17. M. Berndt-Paetz, A. Weimann, N. Sieger, S. Schastak, Y. M. Riyad, J. Griebel, V. K. A. Arthanareeswaran, J. U. Stolzenburg and J. Neuhaus, "Tetrahydroporphyrin-tetratosylat (THPTS): A near-infrared photosensitizer for targeted and efficient photodynamic therapy (PDT) of human bladder carcinoma," Photodiagnosis. Photodyn. Ther. 18, 244-251 (2017).

18. T. Desmettre, J. M. Devoisselle and S. Mordon, "Fluorescence properties and metabolic features of indocyanine green (ICG) as related to angiography," Surv. Ophthalmol. 45, 15-27 (2000).

19. R. Radzi, T. Osaki, T. T. suka, T. Imagama, S. Minami, Y. Nakayama and Y. Okamoto, "Photodynamic hyperthermal therapy with indocyanine green (ICG) induces apoptosis and cell cycle arrest in B16F10 murine melanoma cells," J. Vet. Med. Sci. 74, 545-551 (2012).

20. S. Luo, X. Tan, S. Fang, Y. Wang, T. Liu, X. Wang, Y. Yuan, H. Sun, Q. Qi and C. Shi, "Mitochondriatargeted small-molecule fluorophores for dual modal cancer phototherapy," Adv. Funct. Mater. 26, 2826-2835 (2016). 